\title{
Comparing Interval Management Control Laws for Steady-State Errors and String Stability
}

\author{
Lesley A. Weitz* \\ The MITRE Corporation, McLean, VA, U.S.A. \\ and \\ Kurt A. Swieringa ${ }^{\dagger}$ \\ NASA Langley Research Center, Hampton, VA, U.S.A.
}

\begin{abstract}
Interval Management (IM) is a future airborne spacing concept that leverages avionics to provide speed guidance to an aircraft to achieve and maintain a specified spacing interval from another aircraft. The design of a speed control law to achieve the spacing goal is a key aspect in the research and development of the IM concept. In this paper, two control laws that are used in much of the contemporary IM research are analyzed and compared to characterize steady-state errors and string stability. Numerical results are used to illustrate how the choice of control laws gains impacts the size of steady-state errors and string performance and the potential trade-offs between those performance characteristics.
\end{abstract}

\section{Introduction}

Interval Management (IM) is a future Air Traffic Management concept that uses specialized avionics to provide speed guidance to an aircraft to achieve and maintain a specified spacing interval relative to another aircraft. The IM concept is enabled by the deployment of Automatic Dependent Surveillance-Broadcast (ADS-B), which is a technology that broadcasts aircraft states (i.e., positions and velocities). Aircraft within surveillance range of the broadcasting aircraft can receive this information and use it for advanced flight-deck applications, ranging from situation awareness to providing guidance based on the states of other aircraft.

In the envisioned concept, an air traffic controller, with the help of supporting Air Traffic Control (ATC) automation systems, would identify an aircraft that is equipped with IM avionics (the IM Aircraft), an aircraft from which the IM Aircraft should space (the Target Aircraft), and a spacing goal (the Assigned Spacing Goal). ${ }^{1}$ The IM avionics would then provide speed guidance to achieve and/or maintain the Assigned Spacing Goal relative to the Target Aircraft. Research has shown that IM can provide more precise interaircraft spacing than the use of ATC automation systems alone through the more frequent provision of speed guidance in the presence of uncertainties. ${ }^{2,3}$

Research in flight-deck spacing concepts has been ongoing for a few decades. ${ }^{4}$ A number of speed control algorithms have been developed and tested to support various aspects of the research, including performance analyses using fast-time simulation, human-in-the-loop experiments, and flight tests. ${ }^{5-19}$ The Federal Aviation Administration (FAA) is leading the development of avionics standards and the requisite ATC automation systems to enable IM operations in the U.S. National Airspace System. As a part of the avionics standards effort, a sample IM algorithm has been developed and tested and is described in the recentlypublished Flight-deck Interval Management (FIM) Minimum Operational Performance Standards (MOPS), DO-361. ${ }^{20}$ In addition, the National Aeronautics and Space Administration (NASA) conducted a flight test in 2017 using prototype IM avionics based on the NASA-developed Airborne Spacing for Terminal Arrival Routes (ASTAR) algorithm. The prototype avionics were largely compliant with the avionics standards in DO- $361 .{ }^{21}$

*Principal Systems Engineer, Center for Advanced Aviation System Development, lweitz@mitre.org, AIAA Associate Fellow.

${ }^{\dagger}$ Research Scientist, Crew Systems and Aviation Operations Branch, kurt.a.swieringa@nasa.gov, AIAA Member. 
This paper compares the two IM speed control laws at the core of the spacing algorithms that are being used for much of the contemporary IM research: the Time-to-Go control law in the sample algorithm in DO-361 and NASA's ASTAR control law that was used for the prototype avionics in the recent flight test. While the fundamental design of the control laws is similar, there are differences in design that lead to differences in steady-state errors and string behavior. This comparison is important when aiming to understand differences in performance and how each control law may be tuned to maximize the desired performance (e.g., attenuating spacing errors along a string of aircraft all performing IM operations while also minimizing steady-state errors).

The paper is organized as follows. Additional background on the IM control laws is presented in Section II. The main results are presented in Section III, where the steady-state error and string-stability relationships for each control law are derived using a common framework. Numerical results are used to further compare the two control laws in Section IV, and lastly, conclusions are presented in Section V.

\section{Background}

Two primary stages comprise IM operations: the Achieve Stage and the Maintain Stage. ${ }^{1,16}$ The Achieve Stage defines the part of the IM operation when the IM Aircraft is making progress towards the Assigned Spacing Goal; i.e., the start of the operation until the IM Aircraft crosses the point where the spacing goal is needed, referred to as the Achieve-by Point. The Maintain Stage follows the Achieve Stage. During this part of the IM operation, the speed guidance aims to maintain the spacing interval to within a tolerance of the Assigned Spacing Goal until the IM Aircraft crosses the point where the IM operation is terminated, referred to as the Planned Termination Point. Figure 1 depicts an example IM operation with the Achieve-by Point, Planned Termination Point, and the Achieve and Maintain Stages denoted.

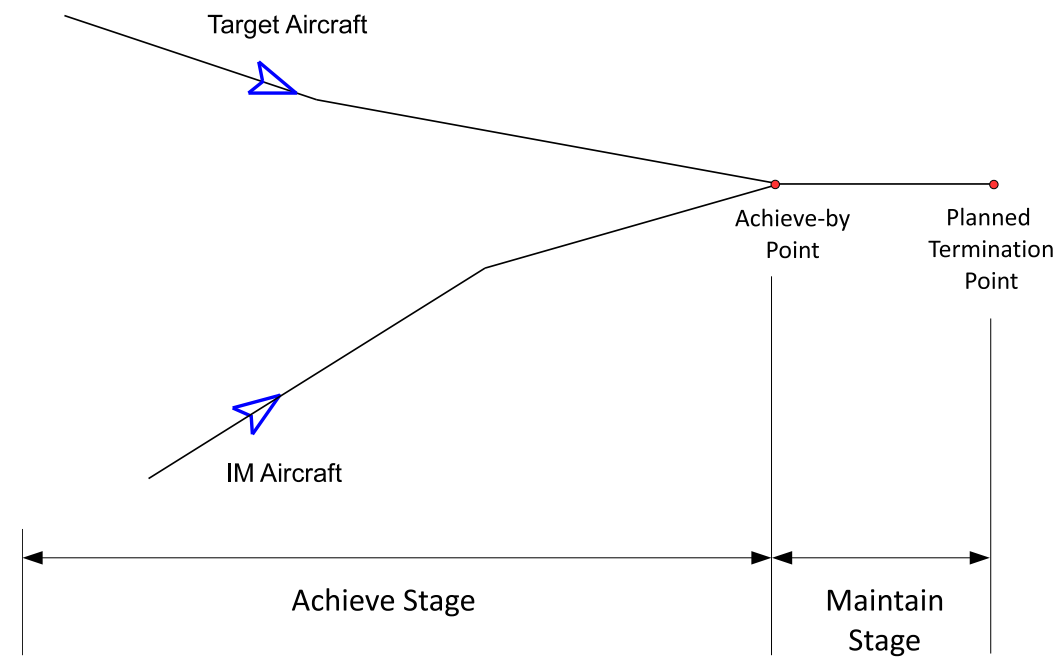

Figure 1. Illustration of an IM Operation when the IM Aircraft is working to achieve the desired spacing interval relative to the Target Aircraft at the Achieve-by Point.

The IM and Target Aircraft are constrained to be on the same route during the Maintain Stage; however, during the Achieve Stage, aircraft may be on different routes. The Achieve-by Point may be located at any point where the routes are common, and as early as the merge point between the routes.

For time-based Assigned Spacing Goals (the primary focus of the majority of IM-related research and this paper), the spacing interval at the Achieve-by Point and during the Maintain Stage is defined as the difference in times that the IM and Target Aircraft cross a common point. Prior to the Achieve-by Point, the spacing is evaluated using the predicted spacing interval, which is the difference in predicted times that the IM and Target Aircraft are expected to cross the Achieve-by Point. The predicted times are derived from four-dimensional (4D) trajectories (i.e., the three dimensional position of the aircraft given as a function of time) generated for both the IM and Target Aircraft.

This paper focuses on speed control laws for the Achieve Stage of IM operations, which provide speed 
guidance to minimize the predicted spacing error at the Achieve-by Point. The predicted 4D trajectories for the IM and Target Aircraft, from which the predicted spacing interval at the Achieve-by Point is determined, are key components of the speed guidance calculation. While this paper does not extensively explore trajectory prediction algorithms, assumptions are made about the reference trajectories to enable the closed-form steady-state error and string-stability analyses in Section III. Further detail on predicting trajectories for the IM and Target Aircraft can be found in references [20] and [22].

There are two primary objectives of the speed control law in the Achieve Stage:

1. the spacing interval at the Achieve-by Point must be within tolerance of the Assigned Spacing Goal, and

2. the speed guidance must be such that spacing errors are attenuated along a string of aircraft performing IM operations.

Relating to the first objective, the tolerance allows some deviation from the Assigned Spacing Goal as a result of any steady-state errors and also due to uncertainties in the environment (e.g., effects of wind conditions). The desired spacing performance at the Achieve-by Point is to achieve spacing errors within 10 seconds of the Assigned Spacing Goal 95\% of the time.

The second objective is related to string stability and the tuning of control law gains to ensure that spacing errors are dissipated along the string, rather than amplified. Weitz and Hurtado provide background and related literature on string stability and how it applies to the IM concept. ${ }^{12}$ The analysis is straightforward for linear systems, which involves determining the error-propagation transfer function that relates spacing errors between adjacent IM pairs in a string. The magnitude of the frequency response function can then be evaluated to see how an input error (i.e., the error in the preceding IM pair) is either amplified or attenuated by the output error (i.e., the next IM pair in the string).

In the IM avionics standards (DO-361), these objectives are represented by performance requirements; however, there is no requirement to implement a particular speed control law. This enables avionics manufacturers to have some flexibility in their design approach. The Time-to-Go control law, which is a part of the sample algorithm provided in DO-361, and the ASTAR control law, which was most recently used to design the prototype IM avionics for the 2017 flight test, are two possible approaches to satisfying the Achieve Stage objectives.

\section{Main Results}

Steady-state error and string stability are examined for the Time-to-Go and ASTAR control laws. For each analysis, it is assumed that a string of aircraft is conducting IM operations. Aircraft 2 through $n+1$ are IM Aircraft spacing relative to their immediately preceding Target Aircraft, resulting in $n$ IM pairs.

\section{A. Simple Aircraft Model}

A single degree-of-freedom, acceleration-level model is used to represent longitudinal aircraft motion and the closed-loop response of the IM Aircraft to speed commands determined by the speed control law.

$$
\dot{x}(t)=v(t) ; \quad \dot{v}(t)=k_{v}\left(v^{c}(t)-v(t)\right)
$$

Here, $x$ is the inertial (along-path) position of the aircraft, and $v$ is the inertial speed. The IM speed control law calculates a speed command, $v^{c}(t)$, and a first-order model is designed to track that speed. The value of the aircraft-performance gain, $k_{v}$, is selected to approximately model the aircraft and its auto-flight system (e.g., autothrottle) dynamics.

\section{B. Predicted Spacing Error}

The predicted spacing error, $e_{i}(t)$, is defined as the difference in the time-to-go (TTG) of the IM Aircraft (the $(i+1)^{t h}$ aircraft) and the TTG of the Target Aircraft (the $i^{t h}$ aircraft) to the Achieve-by Point at time $t$ plus the Assigned Spacing Goal, $\Delta$.

$$
e_{i}(t)=\tau_{i+1}(t)-\left[\tau_{i}(t)+\Delta\right]
$$


Here, $\tau_{i+1}(t)$ and $\tau_{i}(t)$ are the TTGs for the IM and Target Aircraft, respectively.

To enable the closed-form analyses presented here, a constant-speed reference trajectory is assumed with speed $\bar{v}$. Assuming the constant speed, $\bar{v}$, and that the Achieve-by Point is located at along-path position $x_{f}$, the TTG of the $i^{t h}$ aircraft to the Achieve-by Point can be calculated as a function of its along-path position.

$$
\tau_{i}(t)=\frac{x_{f}-x_{i}(t)}{\bar{v}}
$$

Furthermore, the predicted spacing error can now be written as a function of the along-path positions of each aircraft.

$$
e_{i}(t)=\frac{x_{i}(t)-x_{i+1}(t)}{\bar{v}}-\Delta
$$

\section{Time-to-Go Control Law}

The Time-to-Go control law is designed to track the location on the IM Aircraft's reference trajectory where the TTG is equal to the desired TTG, denoted as $\tau_{i+1}^{*}$. The desired TTG is determined such that the predicted spacing error is zero.

$$
\tau_{i+1}^{*}(t)=\tau_{i}(t)+\Delta
$$

The reference TTG is then mapped to an along-path position on the IM Aircraft's reference trajectory, $X_{i+1}\left(\tau_{i+1}^{*}(t)\right)$, from which the reference speed at that along-path position, $\dot{X}_{i+1}\left(\tau_{i+1}^{*}(t)\right)$, may also be determined.

The Time-to-Go control law has the following general form.

$$
v_{i+1}^{c}(t)=\dot{X}_{i+1}\left(\tau_{i+1}^{*}(t)\right)+k\left[X_{i+1}\left(\tau_{i+1}^{*}(t)\right)-x_{i+1}(t)\right]
$$

Here, $k$ is a constant control gain with units $1 / \operatorname{seconds}\left(\sec ^{-1}\right)$.

For a constant-speed reference trajectory, the reference positions and speeds for the $i^{t h}$ aircraft can be written as functions of $\tau_{i}^{*}(t)$.

$$
X_{i}\left(\tau_{i}^{*}(t)\right)=x_{f}-\bar{v} \tau_{i}^{*}(t) \quad \text { and } \quad \dot{X}_{i}\left(\tau_{i}^{*}(t)\right)=\bar{v}
$$

\section{Steady-State Error Analysis}

First, the steady-state error is analyzed for the first IM Aircraft pair (i.e., aircraft 2 following aircraft 1). The first aircraft in the string is assumed to fly speed $v_{1}(t)$, which is not necessarily equal to the assumed reference trajectory speed $\bar{v}$. Taking derivatives of $e_{1}(t)$ yields the following.

$$
\begin{aligned}
e_{1}(t) & =\tau_{2}(t)-\tau_{1}(t)-\Delta=\frac{x_{1}(t)-x_{2}(t)}{\bar{v}}-\Delta \\
\dot{e}_{1}(t) & =\frac{v_{1}(t)-v_{2}(t)}{\bar{v}} \\
\ddot{e}_{1}(t) & =\frac{\dot{v}_{1}(t)-\dot{v}_{2}(t)}{\bar{v}} \\
& =\frac{\dot{v}_{1}(t)}{\bar{v}}-\frac{k_{v}}{\bar{v}}\left[v_{2}^{c}(t)-v_{2}(t)\right] \\
& =\frac{\dot{v}_{1}(t)}{\bar{v}}-\frac{k_{v}}{\bar{v}}\left\{\bar{v}+k\left[x_{f}-\bar{v}\left(\frac{x_{f}-x_{1}(t)}{\bar{v}}+\Delta\right)-x_{2}(t)\right]-v_{2}(t)\right\} \\
& =\frac{\dot{v}_{1}(t)}{\bar{v}}-\frac{k_{v}}{\bar{v}}\left\{\bar{v}\left[1+k e_{1}(t)\right]-v_{2}(t)\right\}
\end{aligned}
$$

Add $-k_{v} / \bar{v} \cdot v_{1}(t)$ to each side of equation (10) to yield the following.

$$
\ddot{e}_{1}(t)+k_{v} \dot{e}_{1}(t)+\left(k_{v} k\right) e_{1}(t)=\frac{1}{\bar{v}} \dot{v}_{1}(t)+\frac{k_{v}}{\bar{v}} v_{1}(t)-k_{v}
$$

The steady-state is reached when $\ddot{e}_{1}(t), \dot{e}_{1}(t)$, and $\dot{v}_{1}(t)$ are equal to zero, which leaves the following relationship.

$$
\left(k_{v} k\right) e_{1, s s}=\frac{k_{v}}{\bar{v}} v_{1, s s}-k_{v}
$$


Solving for $e_{1, s s}$, the steady-state error is

$$
e_{1, s s}=\frac{v_{1, s s}-\bar{v}}{\bar{v} k}
$$

If $v_{1, s s}=\bar{v}+\delta$, then $e_{1, s s}=\frac{\delta}{\bar{v} k}$.

Next examining the spacing of the second through $n^{\text {th }}$ IM Aircraft pairs, the predicted spacing error $e_{i}(t)$ and its derivatives for $i=2, \ldots, n$ are as follows.

$$
\begin{aligned}
& e_{i}(t)=\tau_{i+1}(t)-\tau_{i}(t)-\Delta=\frac{x_{i}(t)-x_{i+1}(t)}{\bar{v}}-\Delta \\
& \dot{e}_{i}(t)= \frac{v_{i}(t)-v_{i+1}(t)}{\bar{v}} \\
& \ddot{e}_{i}(t)= \frac{\dot{v}_{i}(t)-\dot{v}_{i+1}(t)}{\bar{v}}=\frac{k_{v}\left[v_{i}^{c}(t)-v_{i}(t)\right]-k_{v}\left[v_{i+1}^{c}(t)-v_{i+1}(t)\right]}{\bar{v}} \\
&= k_{v}\left[\frac{\bar{v}-v_{i}(t)+k\left(x_{i-1}(t)-x_{i}(t)-\bar{v} \Delta\right)}{\bar{v}}\right]-\ldots \\
& \ldots-k_{v}\left[\frac{\bar{v}-v_{i+1}(t)+k\left(x_{i}(t)-x_{i+1}(t)-\bar{v} \Delta\right)}{\bar{v}}\right]
\end{aligned}
$$

The second-order differential equation relating $e_{i}(t)$ and $e_{i-1}(t)$ is found from equation (16).

$$
\ddot{e}_{i}(t)+k_{v} \dot{e}_{i}(t)+\left(k_{v} k\right) e_{i}(t)=\left(k_{v} k\right) e_{i-1}(t)
$$

In the steady-state, $\ddot{e}_{i}(t)$ and $\dot{e}_{i}(t)$ are equal to zero, which reveals that $e_{i, s s}=e_{i-1, s s}$; i.e., the steady-state error of the $i^{t h}$ aircraft pair is equal to the steady-state error of the $(i-1)^{t h}$ pair.

\section{String-Stability Analysis}

The error-propagation transfer function relates the error of the $i^{\text {th }}$ IM pair to the error of the preceding pair and is found by taking the Laplace Transform of the second-order differential equations derived in the previous section. $H_{1}(s)$ relates the predicted spacing error of the first IM pair to the speed of the first aircraft in the string, and $H_{i}(s)$ relates the predicted spacing errors between adjacent pairs.

$$
H_{1}(s)=\frac{E_{1}(s)}{V_{1}(s)}=\frac{\frac{1}{\bar{v}} s+\frac{k_{v}}{\bar{v}}}{s^{2}+k_{v} s+k_{v} k} \quad \text { and } \quad H_{i}(s)=\frac{E_{i}(s)}{E_{i-1}(s)}=\frac{k_{v} k}{s^{2}+k_{v} s+k_{v} k}
$$

The form of $H_{i}(s)$ is a well-known result from classical control literature. The magnitude of the frequency response function will be less than or equal to one for all input frequencies if the closed-loop damping ratio $\zeta$ is greater than 0.707 , where

$$
\zeta=\sqrt{\frac{k_{v}}{4 k}}
$$

Therefore, good string behavior can be imposed by selecting $k$ such that the closed-loop damping ratio is greater than 0.707 .

$H_{1}(s)$ describes the amplification due to the frequency response of the first aircraft's speed. As it is a function of $\bar{v}$, the results will be explored numerically in Section IV.

\section{ASTAR13 Control Law}

The ASTAR control law analyzed in this paper represents the thirteenth revision, and is also referred to as ASTAR13 in other literature. A key difference in ASTAR13 compared to previous versions of the ASTAR control law is the addition of a groundspeed term, which is the difference between the current speed of the Target Aircraft and the expected speed of the Target Aircraft based on the reference trajectory. ${ }^{10}$ The groundspeed term was added to compensate for cases when the Target Aircraft is flying slower than the expected speed for an extended period of time during the IM operation. Also in contrast to the Time-to-Go control law, the predicted spacing error appears explicitly in the ASTAR control law. 
The ASTAR control law has the following form.

$$
v_{i+1}^{c}(t)=v_{i+1, r e f}+k_{p} e_{i}(t)+k_{G S}\left[v_{i}(t)-v_{i, r e f}\right]
$$

Here, $v_{i+1, r e f}$ and $v_{i, r e f}$ are the reference trajectory speeds at the current positions of the IM Aircraft (aircraft $i+1$ ) and the Target Aircraft (aircraft $i$ ), respectively; $e_{i}(t)$ is the predicted spacing error between the Target Aircraft and the IM Aircraft at the Achieve-by Point; $k_{p}$ is a proportional gain on the predicted spacing error; $k_{G S}$ is the proportional gain for the groundspeed term, and $v_{i}(t)$ is the current speed of the Target Aircraft.

Given the assumption of a constant-speed reference trajectory, the ASTAR control law is simplified.

$$
v_{i+1}^{c}(t)=\bar{v}+k_{p} e_{i}(t)+k_{G S}\left[v_{i}(t)-\bar{v}\right]
$$

\section{Steady-State Error Analysis}

Taking derivatives of $e_{1}(t)$ reveals the second-order differential equation relating $e_{1}(t)$ to the speed of the first aircraft in the string, $v_{1}(t)$.

$$
\begin{aligned}
e_{1}(t) & =\frac{x_{1}(t)-x_{2}(t)}{\bar{v}}-\Delta \\
\dot{e}_{1}(t) & =\frac{v_{1}(t)-v_{2}(t)}{\bar{v}} \\
\ddot{e}_{1}(t) & =\frac{\dot{v}_{1}(t)-\dot{v}_{2}(t)}{\bar{v}} \\
& =\frac{\dot{v}_{1}(t)}{\bar{v}}-\frac{k_{v}}{\bar{v}}\left[v_{2}^{c}(t)-v_{2}(t)\right] \\
& =\frac{\dot{v}_{1}(t)}{\bar{v}}-\frac{k_{v}}{\bar{v}}\left[\bar{v}+k_{p} e_{1}(t)+k_{G S}\left(v_{1}(t)-\bar{v}\right)-v_{2}(t)\right]
\end{aligned}
$$

Adding $-k_{v} / \bar{v} \cdot v_{1}(t)$ to both sides of equation (24) results in the following.

$$
\ddot{e}_{1}(t)+k_{v} \dot{e}_{1}(t)+\left(\frac{k_{v} k_{p}}{\bar{v}}\right) e_{1}(t)=\frac{1}{\bar{v}} \dot{v}_{1}(t)+\frac{k_{v}}{\bar{v}}\left(1-k_{G S}\right) v_{1}(t)-k_{v}\left(1-k_{G S}\right)
$$

The steady-state error is analyzed in the time domain. The following expression reveals the steady-state error $e_{1, s s}$ when $\dot{e}_{1}(t)=\ddot{e}_{1}(t)=\dot{v}_{1}(t)=0$.

$$
\left(\frac{k_{v} k_{p}}{\bar{v}}\right) e_{1, s s}=\frac{k_{v}}{\bar{v}}\left(1-k_{G S}\right) v_{1, s s}-k_{v}\left(1-k_{G S}\right)
$$

Solving for $e_{1, s s}$, yields the following

$$
e_{1, s s}=\frac{\left(1-k_{G S}\right) v_{1, s s}-\bar{v}\left(1-k_{G S}\right)}{k_{p}} .
$$

Setting $v_{1, s s}=\bar{v}+\delta, e_{1, s s}=\frac{\left(1-k_{G S}\right) \delta}{k_{p}}$.

The second-order differential equation relating $e_{i}(t)$ to $e_{i-1}(t)$ for $i=2, . ., n$ is revealed by taking derivatives of $e_{i}(t)$.

$$
\begin{aligned}
e_{i}(t) & =\frac{x_{i}(t)-x_{i+1}(t)}{\bar{v}}-\Delta \\
\dot{e}_{i}(t) & =\frac{v_{i}(t)-v_{i+1}(t)}{\bar{v}} \\
\ddot{e}_{i}(t) & =\frac{\dot{v}_{i}(t)-\dot{v}_{i+1}(t)}{\bar{v}} \\
& =\frac{k_{v}}{\bar{v}}\left[v_{i}^{c}(t)-v_{i}(t)\right]-\frac{k_{v}}{\bar{v}}\left[v_{i+1}^{c}(t)-v_{i+1}(t)\right] \\
& =\frac{k_{v}}{\bar{v}}\left[\bar{v}+k_{p} e_{i}(t)+k_{G S}\left(v_{i-1}(t)-\bar{v}\right)-v_{i}(t)\right]-\frac{k_{v}}{\bar{v}}\left[\bar{v}+k_{p} e_{i+1}(t)+k_{G S}\left(v_{i}(t)-\bar{v}\right)-v_{i+1}(t)\right]
\end{aligned}
$$


Rearranging equation (30) results in the following relationship.

$$
\ddot{e}_{i}(t)+k_{v} \dot{e}_{i}(t)+\frac{k_{v} k_{p}}{\bar{v}} e_{i}(t)=k_{v} k_{G S} \dot{e}_{i-1}(t)+\frac{k_{v} k_{p}}{\bar{v}} e_{i-1}(t)
$$

When the time derivatives are equal to zero in equation (31), $e_{i, s s}=e_{i-1, s s}$.

\section{String-Stability Analysis}

Again, the error-propagation transfer functions are determined by taking the Laplace transform of equations (25) and (31).

$$
H_{1}(s)=\frac{E_{1}(s)}{V_{1}(s)}=\frac{\frac{1}{\bar{v}} s+\frac{k_{v}}{\bar{v}}\left(1-k_{G S}\right)}{s^{2}+k_{v} s+\frac{k_{v} k_{p}}{\bar{v}}} \quad \text { and } \quad H_{i}(s)=\frac{E_{i}(s)}{E_{i-1}(s)}=\frac{k_{v} k_{G S} s+\frac{k_{v} k_{p}}{\bar{v}}}{s^{2}+k_{v} s+\frac{k_{v} k_{p}}{\bar{v}}}
$$

It should be noted that the key differences in the error-propagation transfer functions for the Time-to-Go and ASTAR control laws are the $s^{0}$ coefficient in the characteristic equation and the inclusion of the $k_{G S}$ parameter in the numerators of $H_{1}(s)$ and $H_{i}(s)$.

\section{Numerical Results}

Numerical results for different parameter values are presented to further compare the steady-state errors and string-stability properties for each control law.

\section{A. Steady-State Errors}

The steady-state error for the Time-to-Go control law is a function of control law gain $k$, the assumed constant speed of the reference trajectory $\bar{v}$, and the Target Aircraft's deviation from the reference trajectory speed, $\delta$. Figure 2 shows the steady-state errors as a function of $\delta$ and $\bar{v}$ for four different values of $k: k=0.005$, $0.008,0.015$, and $0.030 \mathrm{sec}^{-1}$. As shown in Figure 2, the steady-state errors are smaller as the value of $k$ increases and as the speed of the reference trajectory increases.

The ASTAR control law is a function of control law gain $k_{p}$, the groundspeed gain $k_{G S}$, and $\delta$. In past research, the groundspeed gain has been given a value of 1 until the IM Aircraft is within 40 nautical miles (nmi) of the Achieve-by Point, where the groundspeed gain is linearly decreased to zero (at a distance of $20 \mathrm{nmi}$ to the Achieve-by Point). The groundspeed term is also inhibited when the Target Aircraft's groundspeed is faster than predicted. The primary reason for these modifications to the groundspeed gain is to reduce the number of undesirable speed increases, particularly when the aircraft is close to the runway. For the purposes of this paper, the groundspeed gain is assumed to be given a value of 1 or 0 (i.e., "on" or "off"). When the groundspeed term is "on," $k_{p}$ has a value of $0.375 \mathrm{knot}(\mathrm{kt}) / \mathrm{sec}$. This value increases linearly as the distance to the Achieve-by Point decreases, reaching a value around $1.000 \mathrm{kt} / \mathrm{sec}$ when $k_{G S}$ becomes 0 , before reaching a constant value of 1.5 or $2.000 \mathrm{kt} / \mathrm{sec}$.

Figure 3 shows the steady-state errors for the ASTAR control law as a function of $\delta, k_{p}$, and $k_{G S}\left(k_{G S}=0\right.$ is on the left and $k_{G S}=1$ is on the right). Four values of $k_{p}$ are shown in each plot: $k_{p}=0.375,1.000,1.500$, and $2.000 \mathrm{kt} / \mathrm{sec}^{\mathrm{a}}{ }^{\mathrm{T}}$ The steady-state errors are all zero (for all values of $k_{p}$ ) in the case that the groundspeed term is "on." In the case that $k_{G S}$ is "off," the steady-state errors decrease with larger values of $k_{p}$. As noted in the previous paragraph, $k_{p}$ is expected to be between 1.000 and $2.000 \mathrm{kt} / \mathrm{sec}$ when $k_{G S}=0$; therefore, the steady-state errors will be 20 seconds or less when the Target Aircraft is slower than the reference trajectory speed by as much as $20 \mathrm{kt}$.

It should be noted that the IM avionics standard requires the IM speed guidance to be limited to $\pm 15 \%$ of the reference trajectory speed. The bounds on the speeds are intended to prevent unreasonable speeds from being presented to the flight crew. It is also expected that the first aircraft's deviation from the assumed reference trajectory will be bounded to a range of reasonable speeds, therefore bounding the steady-state errors.

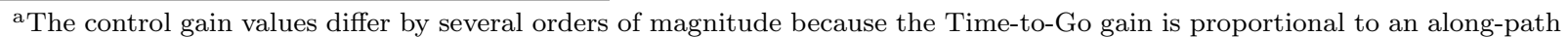
distance error ( $k$ has units of $\left.\sec ^{-1}\right)$, whereas the ASTAR gain is proportional to the predicted spacing error itself $\left(k_{p}\right.$ has units of $\mathrm{kt} / \mathrm{sec})$.
} 

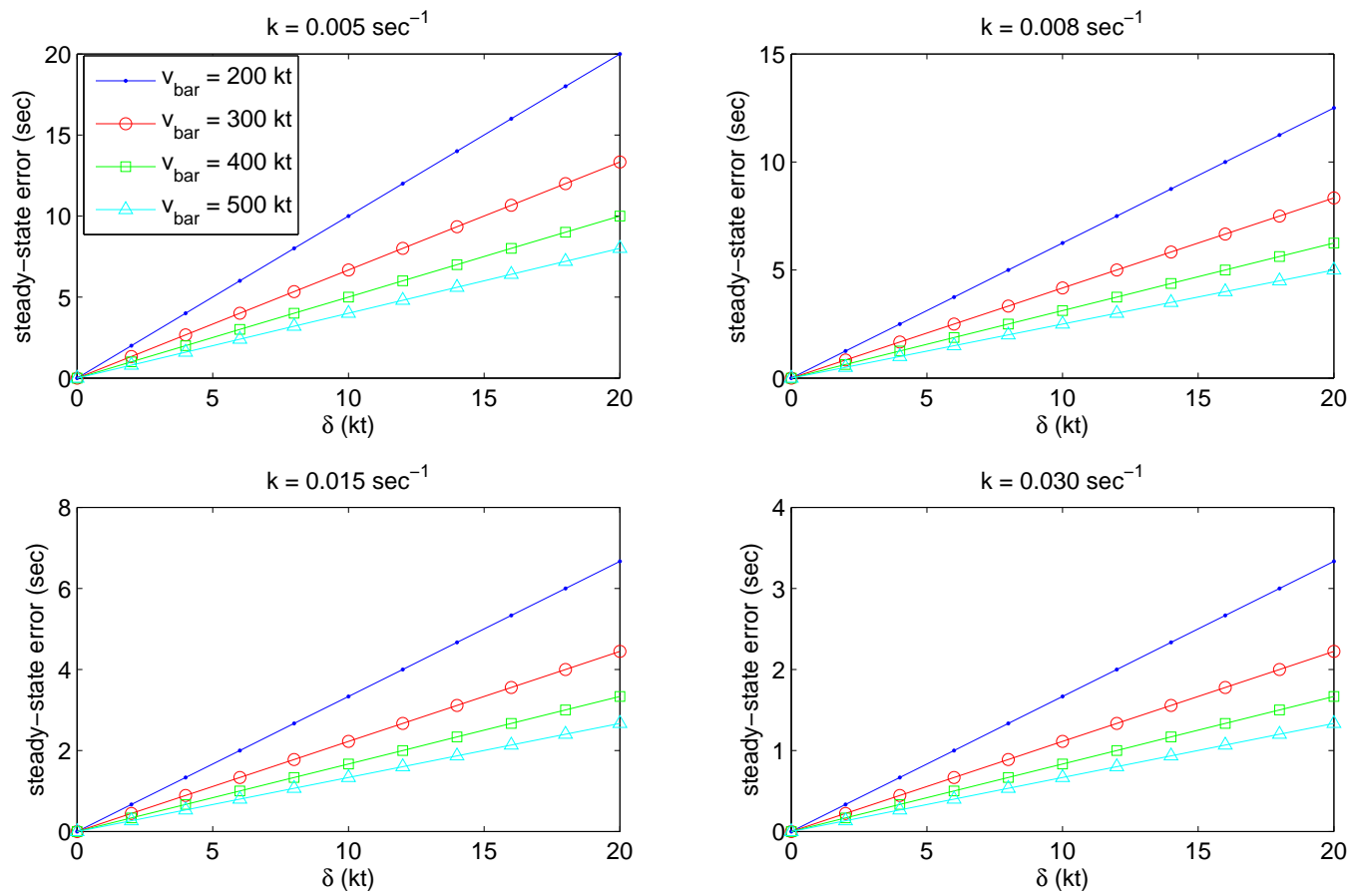

Figure 2. Time-to-Go control law: steady-state errors as a function of $\delta, \bar{v}$, and $k$.
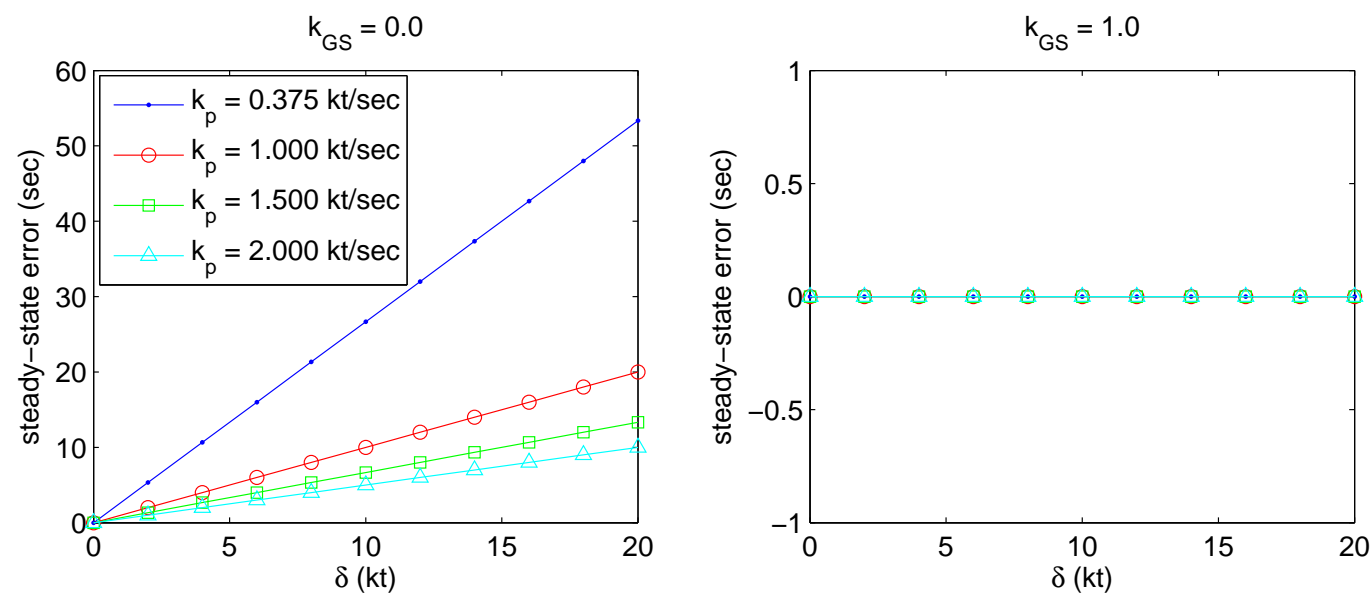

Figure 3. ASTAR control law: steady-state errors as a function of $\delta, k_{p}$, and $k_{G S}$. 


\section{B. String Stability}

As described previously, string stability is evaluated using the frequency-response magnitudes of the errorpropagation transfer functions presented in the previous section.

For the Time-to-Go control law, $H_{1}(s)$, which relates the spacing error for the first IM pair to the speed of the first aircraft in the string, is a function of the aircraft performance gain $k_{v}$, the control law gain $k$, and the speed of the reference trajectory $\bar{v}$. Results show little effect from the control gain $k$, as the magnitudes are grouped by values of $k_{v}$ (i.e., the solid, dashed, and dash-dot lines are overlaid in each of the subplots). The magnitudes also decrease with increased values of $\bar{v}$.
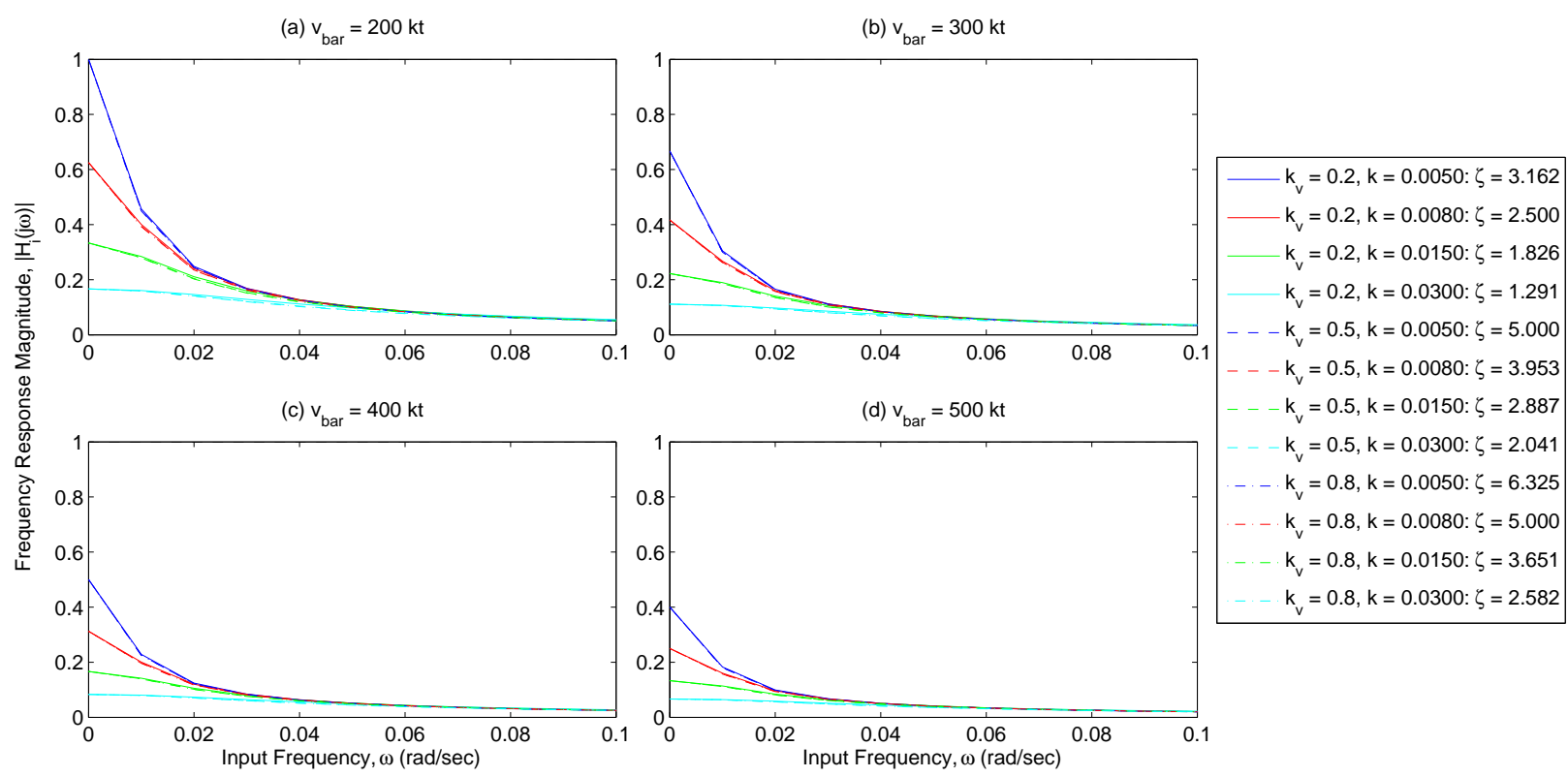

Figure 4. Time-to-Go control law: frequency-response magnitudes for $H_{1}(s)$ with different values of $k_{v}, k$, and $\bar{v}$.

Also described in Section III, the magnitudes of $H_{i}(s)$ will be less than or equal to one if the closed-loop damping ratio is greater than 0.707. This result is shown in Figure 5 for four values of $\zeta: \zeta=0.600,0.707$, 1.000 , and 2.000, and as a function $k_{v}$. As expected, Figure 5 shows that for damping ratios less than 0.707 (the dark blue lines in the figure), the frequency response function will be greater than one for a range of input frequencies. Therefore, the spacing errors will be amplified given inputs with frequencies in that range.

Figure 6 shows the frequency-response magnitudes using the same gain values as shown in Figure 2. In each case, the closed-loop damping ratios are greater than 0.707 and the magnitudes are less than one for all input frequencies. As expected, input errors will be attenuated more quickly with greater damping ratios. However, there is a trade-off that must be considered when selecting the gain value. While larger damping ratios will more quickly attenuate spacing errors along the string, those gains may not provide sufficient response to achieve the desired tolerance at the Achieve-by Point in the presence of uncertainties.

The frequency-response magnitudes of $H_{1}(s)$ for the ASTAR control law are shown in Figures 7 and 8 for $k_{G S}=0$ and 1, respectively. When $k_{G S}=0$, the results are largely driven by the values of $k_{v}$ and $\bar{v}$ with little effect due to the value of $k_{p}$. When $k_{G S}=1$, the $s^{0}$ coefficient in the numerator of $H_{1}(s)$ goes to zero, and the effects of $k_{v}$ and $k_{p}$ are revealed.

Figures 9 and 10 show the frequency-response magnitudes for $H_{i}(s)$ when $k_{G S}=0$ and 1 , respectively. Figure 9 shows that the ASTAR control law leads to string stability for all values of $k_{v}, k_{p}$, and $\bar{v}$ as all magnitudes are less than or equal to one for all input frequencies. Furthermore, the impact of $\bar{v}$ on the dissipation of spacing errors along the string is minimal. However, Figure 10 shows that the ASTAR control law will propagate spacing errors with input frequencies in a small range when $k_{G S}=1$. For the parameter values studied here, the worst case is for $k_{v}=0.2$ (slowest aircraft response), $k_{p}=2.0 \mathrm{kt} / \mathrm{sec}$ (largest control gain), and $\bar{v}=200 \mathrm{kt}$ (slowest reference speed). For that set of values, the maximum frequency-response magnitude is 1.04 , which means that there will be a $4 \%$ increase in the spacing error magnitudes along the 


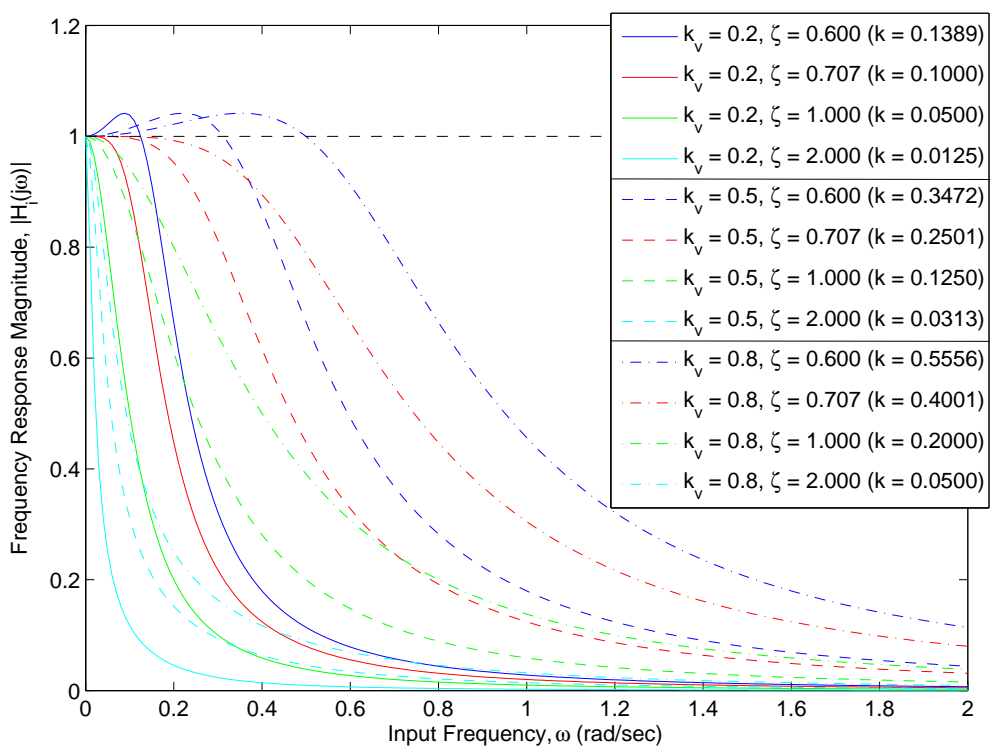

Figure 5. Time-to-Go control law: frequency-response magnitudes for $H_{i}(s)$ with different values of $k_{v}$ and closed-loop damping ratio $\zeta$.

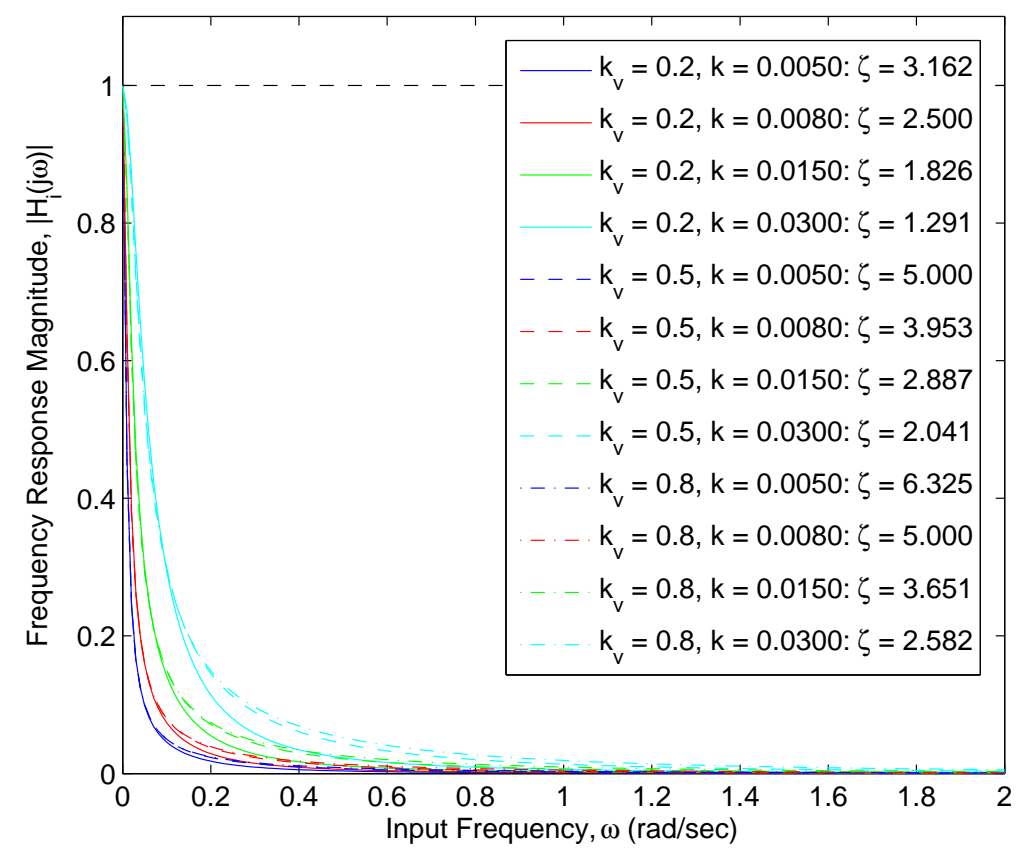

Figure 6. Time-to-Go control law: frequency-response magnitudes for $H_{i}(s)$ with different values of $k_{v}$ and $k$. 
(a) $v_{\text {bar }}=200 \mathrm{kt}$

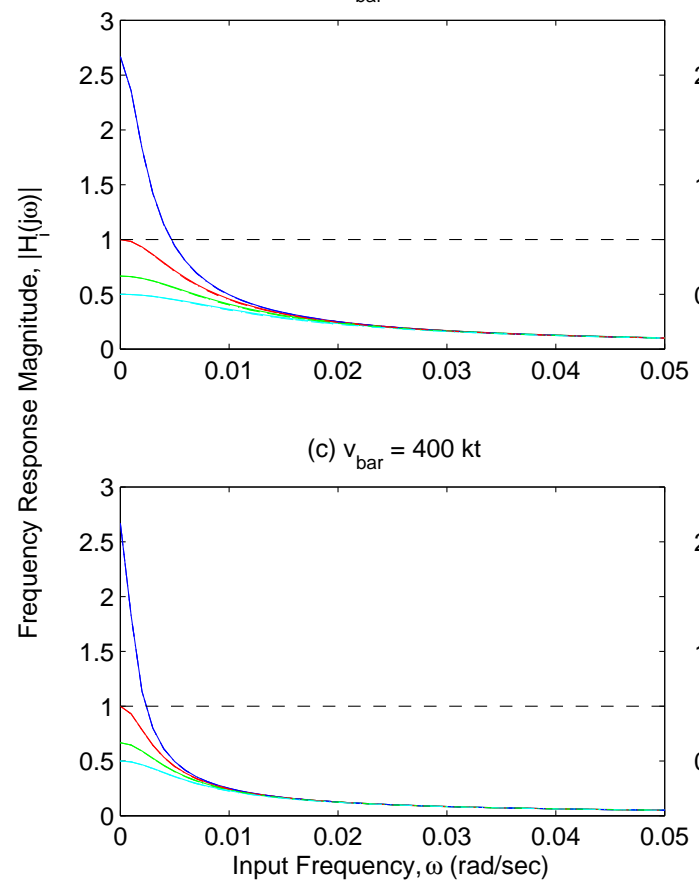

(b) $v_{\text {bar }}=300 \mathrm{kt}$

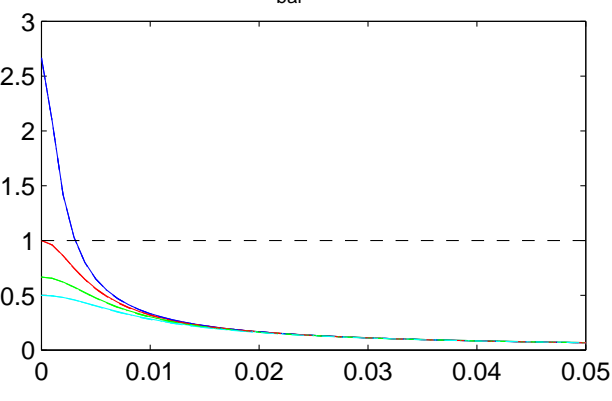

(d) $v_{b a r}=500 \mathrm{kt}$

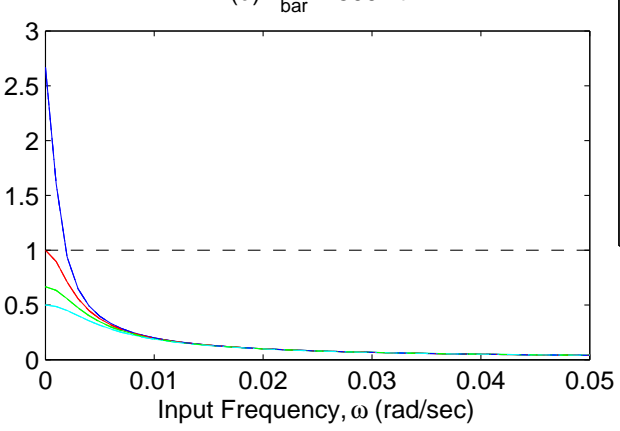

$-\mathrm{k}_{\mathrm{v}}=0.2, \mathrm{k}_{\mathrm{p}}=0.375$ $\mathrm{k}_{\mathrm{v}}=0.2, \mathrm{k}_{\mathrm{p}}=1.000$ $\mathrm{k}_{\mathrm{v}}=0.2, \mathrm{k}_{\mathrm{p}}=1.500$ $\begin{aligned} k_{v} & =0.2, k_{p}=2.000 \\ --k_{v} & =0.5, k_{p}=0.375\end{aligned}$ $--k_{v}=0.5, k_{p}=1.000$ $\mathrm{k}_{\mathrm{v}}=0.5, \mathrm{k}_{\mathrm{p}}=1.500$ $\mathrm{k}_{\mathrm{v}}=0.5, \mathrm{k}_{\mathrm{p}}=2.000$ -.. $\mathrm{k}_{\mathrm{v}}=0.8, \mathrm{k}_{\mathrm{p}}=0.375$ $\cdots \mathrm{k}_{\mathrm{v}}=0.8, \mathrm{k}_{\mathrm{p}}=1.000$ $\ldots \mathrm{k}_{\mathrm{v}}=0.8, \mathrm{k}_{\mathrm{p}}=1.500$ $k_{v}=0.8, k_{p}=2.000$

Figure 7. ASTAR control law: frequency-response magnitudes for $H_{1}(s)$ with different values of $k_{v}$, $k_{p}$, and $\bar{v}$, and for $k_{G S}=0$.

(a) $v_{\text {bar }}=200 \mathrm{kt}$
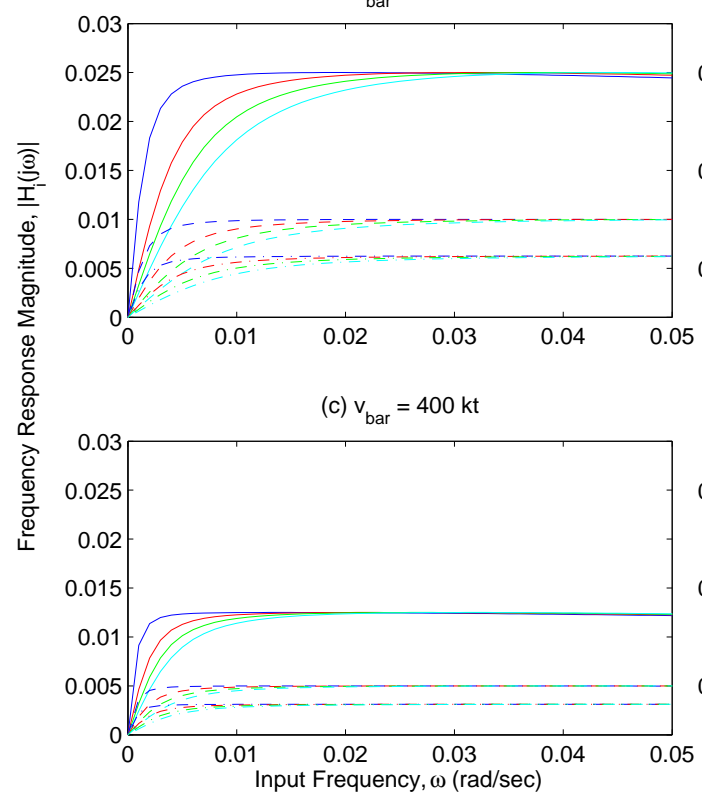

(b) $v_{\text {bar }}=300 \mathrm{kt}$

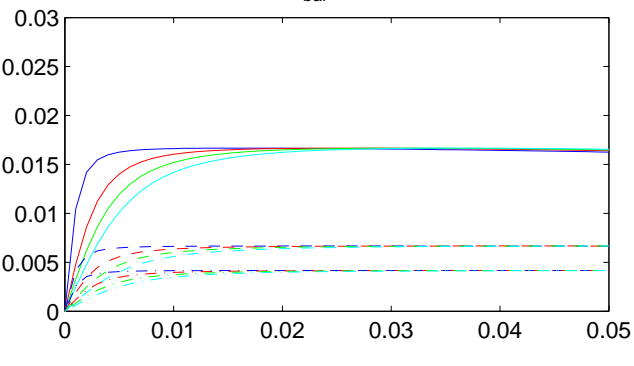

(d) $v_{\text {bar }}=500 \mathrm{kt}$

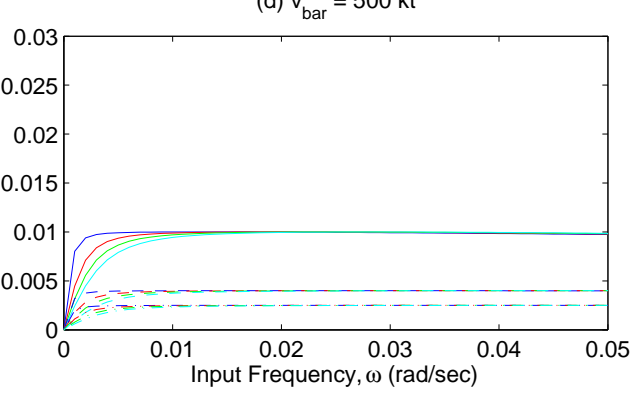

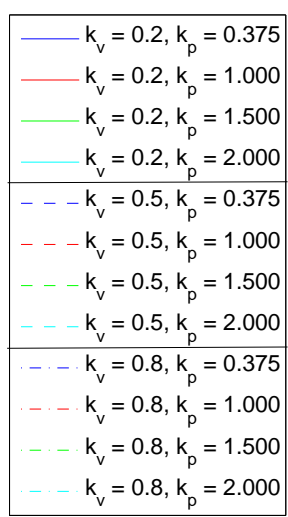

Figure 8. ASTAR control law: frequency-response magnitudes for $H_{1}(s)$ with different values of $k_{v}, k_{p}$, and $\bar{v}$, and for $k_{G S}=1$. 
string. It should be noted that this increase is relatively small and may be managed by limiting the length of aircraft strings or through limits on the speed guidance relative to the reference trajectory speed.

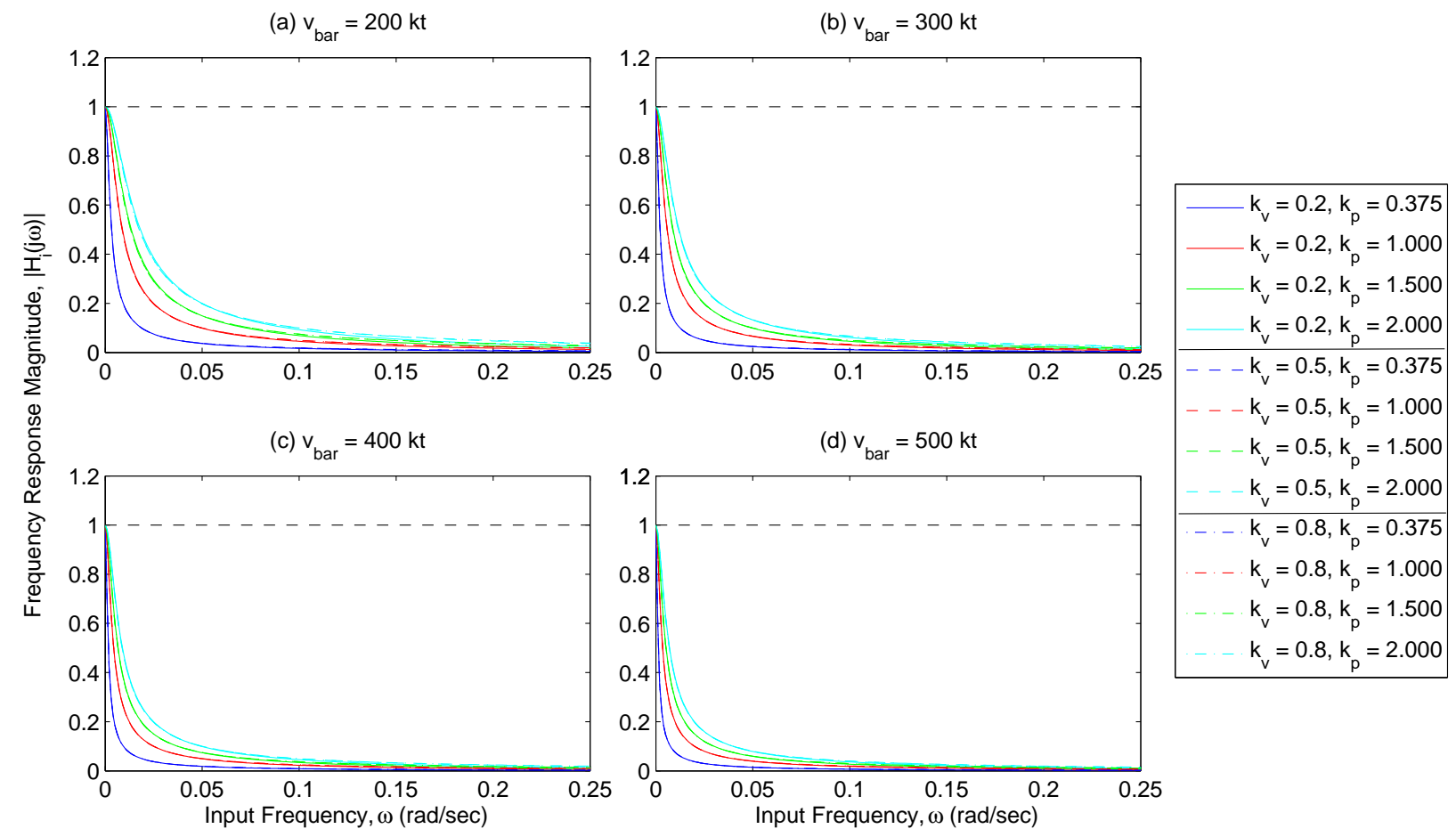

Figure 9. ASTAR control law: frequency-response magnitudes for $H_{i}(s)$ with different values of $k_{v}, k_{p}$, and $\bar{v}$, and for $k_{G S}=0$.

\section{Conclusions}

This paper compared steady-state errors and string stability for two Interval Management (IM) speed control laws that have been used in recent IM research activities.

The Time-to-Go control law, which is a key component of the sample algorithm included in the IM avionics standards, will have steady-state errors if the first aircraft in a string of IM operations deviates from the expected speed in the reference trajectory. The steady-state error increases as a function of the Target Aircraft's deviation from the expected speed and is decreased with larger values of the control law gain. Good string behavior (i.e., spacing errors will be dissipated along the string) is achieved for closed-loop damping ratios greater than 0.707 .

The ASTAR control law, which was the basis for the prototype IM avionics in a recent flight test, includes a groundspeed feedback term for cases when the Target Aircraft deviates from its expected speed in the reference trajectory for an extended duration of the IM operation. In this paper, the gain for the groundspeed was set to either 0 or 1 , representing the states when the groundspeed feedback term is either "off" or "on," respectively. When this term is "on," the steady-state errors are zero for all choices of control gain; however, this causes some undesired string behavior when the frequency-response magnitudes exceed one for a range of input frequencies. In contrast, when the groundspeed term is "off," the steady-state errors are non-zero, but frequency-response magnitudes are less than one for all choices of the control gain and input frequencies. These results reveal the trade-offs in choosing control gains to minimize steady-state errors and ensure good string behavior.

This paper will help inform future speed control law design for IM, including the selection of control gains to balance the size of steady-state errors and string behavior. 
(a) $v_{b a r}=200 \mathrm{kt}$

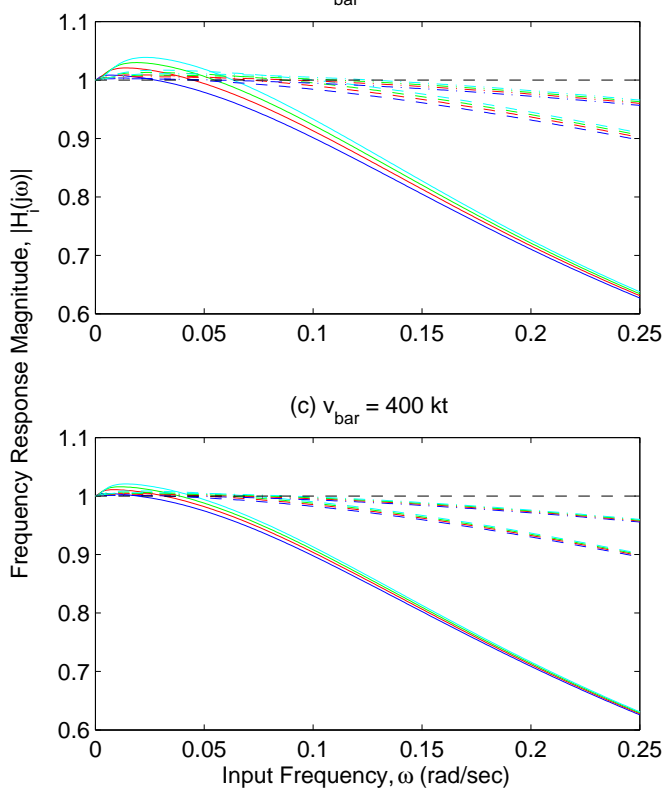

(b) $v_{\text {bar }}=300 \mathrm{kt}$

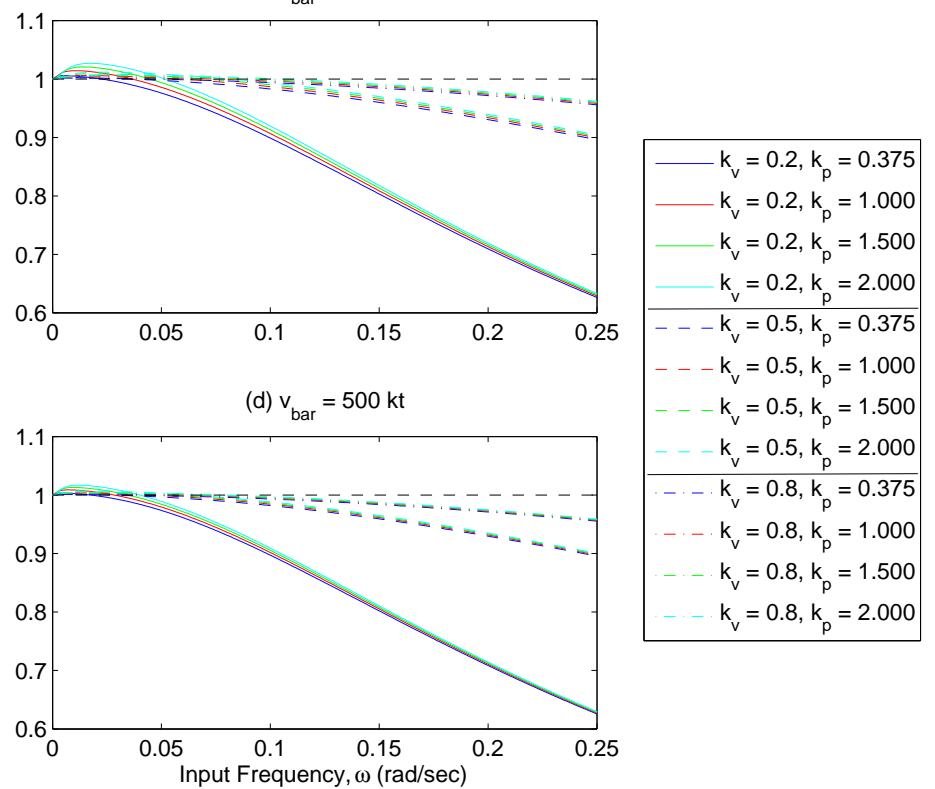

Figure 10. ASTAR control law: frequency-response magnitudes for $H_{i}(s)$ with different values of $k_{v}$, $k_{p}$, and $\bar{v}$, and for $k_{G S}=1$.

\section{References}

${ }^{1}$ Hicok, D. S. and Barmore, B. E., "Concept of Operations for Interval Management Arrivals and Approach," AIAA Guidance, Navigation, and Control Conference, 2016.

${ }^{2}$ Weitz, L. A., Katkin, R., Moertl, P., Penhallegon, W. J., Hammer, J. B., Bone, R. S., and Peterson, T., "Considerations for Interval Management Operations in a Mixed-Equipage Environment," AIAA Aviation Technology, Integration, and Operations Conference, 2012.

${ }^{3}$ Swieringa, K. A., Wilson, S. R., and Baxley, B. T., "System Performance of an Integrated Airborne Spacing Algorithm with Ground Automation," AIAA Aviation Technology, Integration, and Operations Conference, 2016.

${ }^{4}$ Barmore, B. E., Penhallegon, W. J., Weitz, L. A., Bone, R. S., Levitt, I., Flores-Kriegsfeld, J. A., Arbuckle, D., and Johnson, W. C., "Interval Management: Development and Implementation of an Airborne Spacing Concept," AIAA Guidance, Navigation, and Control Conference, 2016.

${ }^{5}$ Hoffman, E., Ivanescu, D., Shaw, C., and Zeghal, K., "Analysis of Constant Time Delay Airborne Spacing Concepts Between Aircraft of Mixed Types in Varying Wind Conditions," Proceedings of the 5th USA/Europe Air Traffic Management R\&D Seminar, Budapest, Hungary, 2003.

${ }^{6}$ Ivanescu, D., Shaw, C., Hoffman, E., and Zeghal, K., "Towards Performance Requirements for Airborne Spacing A Sensitivity Analysis of Spacing Accuracy," Proceedings of the AIAA Aviation Technology, Integration, and Operations Conference, 2006.

${ }^{7}$ Itoh, E., Everdij, M., Bakker, B., and Blom, H., "Speed Control for Airborne Separation Assistance in Continuous Descent Arrivals," Proceedings of the AIAA Aviation Technology Integration and Operations Conference, 2009.

${ }^{8}$ Abbott, T. S., "A Brief History of Airborne Self-Spacing Concepts," Tech. rep., NASA Langley Research Center, Hampton, VA, February 2009, NASA/CR2009-215695.

${ }^{9}$ Barmore, B. E., "Airborne precision spacing: a trajectory-based approach to improve terminal area operations," Digital Avionics and Systems Conference, 2006.

${ }^{10}$ Barmore, B. E., Swieringa, K. A., Underwood, M., Abbott, T., and Leonard, R. D., "Development of an Interval Management Algorithm using Ground Speed Feedback for Delayed Traffic," AIAA Guidance, Navigation, and Control Conference, 2016.

${ }^{11}$ Weitz, L. A. and Hurtado, J. E., "A Time-to-Go Control Law for Spacing Vehicles to a Point," Proceedings of the AIAA Guidance, Navigation, and Control Conference, 2011.

${ }^{12}$ Weitz, L. A. and Hurtado, J. E., "String Stability Analysis of Selected Speed Control Laws for Interval Management," AIAA Guidance, Navigation, and Control Conference, 2012.

${ }^{13}$ Weitz, L. A. and Stassen, H., "A New Spacing Algorithm to Support Near-Term Interval Management Operations," AIAA Aviation Forum, June 2014.

${ }^{14}$ Lohr, G. W., Oseguera-Lohr, R. M., Abbott, T. S., Capron, W. R., and Howell, C. T., "Airborne Evaluation and Demonstration of a Time-based Airborne Inter-arrival Spacing Tool," Tech. rep., NASA Langley Research Center, Hampton, VA, December 2005, NASA/CR2005-213772. 
${ }^{15}$ Penhallegon, W. J., Bone, R. S., and Stassen, H. P., "Results from a Field Evaluation of Interval Management during an Optimized Profile Descent Arrival and Approach," AIAA Guidance, Navigation, and Control Conference, 2016.

${ }^{16}$ RTCA, "Safety, Performance and Interoperability Requirements Document for Airborne Spacing Flight-deck Interval Management (ASPA-FIM)," DO-328A/ED-195, November 2015.

${ }^{17}$ Thipphavong, J., Jung, J., Swenson, H. N., Witzberger, K. E., Lin, M. I., Nguyen, J., Martin, L., Downs, M. B., and Smith, T. A., "Evaluation of the Controller-Managed Spacing Tools, Flight-deck Interval Management and Terminal Area Metering Capabilities for the ATM Technology Demonstration \#1," Proceedings of the USA/Europe Air Traffic Management RED Seminar, Chicago, Illinois, 2013.

${ }^{18}$ Wilson, S. R., Kibler, J. L., Hubbs, C. E., and Smail, J. W., "Air Traffic Management Technology Demonstration1 Research and Procedural Testing of Routes," Tech. rep., NASA Langley Research Center, Hampton, VA, May 2015, NASA/CR2015-218707.

${ }^{19}$ Roper, R. D., "ATD-1 EcoDemonstrator ASTAR Guided Arrival Research (EAGAR)," NASA Briefing, Document ID 20160006919 (available on ntrs.nasa.gov).

${ }^{20}$ RTCA, "Minimum Operational Performance Standards (MOPS) for Flight-deck Interval Management (FIM)," DO-361, June 2015.

${ }^{21}$ Swieringa, K. A., Wilson, S. R., Baxley, B. T., Roper, R. D., Abbott, T. S., Levitt, I., and Scharl, J., "Flight Test Evaluation of the ATD-1 Interval Management Application," AIAA Aviation Technology, Integration, and Operations Conference, 2017.

${ }^{22}$ Abbott, T. S., "A Trajectory Algorithm to Support En Route and Terminal Area Self-Spacing Concepts: Third Revision," Tech. rep., NASA Langley Research Center, Hampton, VA, July 2014, NASA/CR2014-218288. 